PROCEEDINGS OF THE AMERICAN MATHEMATICAL SOCIETY

Volume 135, Number 5, May 2007, Pages 1377-1388

S 0002-9939(06)08583-2

Article electronically published on October 18, 2006

\title{
TRACE CLASS CRITERIA FOR BILINEAR HANKEL FORMS OF HIGHER WEIGHTS
}

\author{
MARCUS SUNDHÄLL
}

(Communicated by Joseph A. Ball)

\begin{abstract}
In this paper we give a complete characterization of higher weight Hankel forms, on the unit ball of $\mathbb{C}^{d}$, of Schatten-von Neumann class $\mathcal{S}_{p}, 1 \leq$ $p \leq \infty$. For this purpose we give an atomic decomposition for certain Besovtype spaces. The main result is then obtained by combining the decomposition and our earlier results.
\end{abstract}

\section{INTRODUCTION}

Hankel operators on the unit disc have been studied extensively; see [Pe1] for a systematic treatment. One of the main topics is to study Schatten-von Neumann properties of Hankel operators; see [Pe1] and [Pe2]. In [JP] Janson and Peetre introduced Hankel forms of higher weights on the unit disc. Their Schatten-von Neumann properties were studied in [Ro and [Z].

In P1 Peetre introduced Hankel forms of higher weights on the unit ball in $\mathbb{C}^{d}$. Their Schatten-von Neumann, $\mathcal{S}_{p}$, properties were studied in $\mathrm{Su}$ for $2 \leq p \leq \infty$. See also [FR] for a different approach.

The results for $2 \leq p \leq \infty$ in [Su] were proved by using interpolation between $\mathcal{S}_{2}$ and $\mathcal{S}_{\infty}$ (bounded operators) and boundedness of certain matrix-valued Bergman projections, but the case of $1 \leq p<2$ was left open there.

In this paper we extend the results in $[\mathrm{Su}$. to $1 \leq p \leq \infty$. For this purpose we study the atomic decomposition for some Besov spaces of vector-valued holomorphic functions, see Section 4 , which then gives $\mathcal{S}_{1}$ properties. Our results follow by interpolation, and we get a full characterization for $1 \leq p \leq \infty$. Some of the proofs in this paper are based on techniques used in $\underline{\mathrm{Su}}$ and will therefore be given briefly. The reader is referred to that article for more details.

The paper is organized as follows. In section 2 we recall briefly some notation and we prove Theorem [2.1, generalizing the result for $p=2$ in $\mathrm{Su}$. Section 3 is devoted to duality relations for the spaces of symbols. In Section 4 we give an atomic decomposition for a certain space of symbols, which will be used in Section 5 to prove the $\mathcal{S}_{1}$ criterion.

Received by the editors September 26, 2005 and, in revised form, November 22, 2005.

2000 Mathematics Subject Classification. Primary 32A25, 32A36, 32A37, 47B32, 47B35.

Key words and phrases. Hankel forms, Schatten-von Neumann classes, Bergman spaces, Bergman projections, duality of Besov spaces, atomic decomposition, transvectants, unitary representations, Möbius group.

This work is part of the author's ongoing Ph.D. thesis under the supervision of Yang Liu and Genkai Zhang. He would like to thank Örebro University for the financial support.

(C)2006 American Mathematical Society 1377

Reverts to public domain 28 years from publication 


\section{Preliminaries}

2.1. The Banach space $\mathcal{H}_{\nu, s}^{p}$ for $1 \leq p \leq \infty$. Let $d m$ denote the Lebesgue measure on the unit ball $\mathbb{B} \subset \mathbb{C}^{d}$ and let $d \iota(z)$ be the measure $\left(1-|z|^{2}\right)^{-d-1} d m(z)$. For $d<\nu<\infty$ let $d \iota_{\nu}(z)$ be the measure $c_{\nu}\left(1-|z|^{2}\right)^{\nu} d \iota(z)$, where $c_{\nu}$ is chosen such that

$$
\int_{\mathbb{B}} d \iota_{\nu}(z)=1
$$

The closed subspace of all holomorphic functions in $L^{2}\left(d \iota_{\nu}\right)$ is denoted by $L_{a}^{2}\left(d \iota_{\nu}\right)$ and is called a weighted Bergman space. Note that the space $L_{a}^{2}\left(d \iota_{\nu}\right)$ has a reproducing kernel $K_{z}(w)=(1-\langle w, z\rangle)^{-\nu}$, that is,

$$
f(z)=\left\langle f, K_{z}\right\rangle_{\nu}=\int_{\mathbb{B}} f(w) \overline{K_{z}(w)} d \iota_{\nu}(w), \quad f \in L_{a}^{2}\left(d \iota_{\nu}\right), \quad z \in \mathbb{B} .
$$

Denote by $B(z, w)$ the Bergman operator on $V=\mathbb{C}^{d}$ as in $[\mathbf{L}$, namely

$$
B(z, w)=(1-\langle z, w\rangle)\left(I-z \otimes w^{*}\right),
$$

where $z \otimes w^{*}$ stands for the rank one operator given by $\left(z \otimes w^{*}\right)(v)=\langle v, w\rangle z$.

The Bergman metric at $z \in \mathbb{B}$, when we identify the tangent space with $V$, is $\left\langle B(z, z)^{-1} u, v\right\rangle$ for $u, v \in V$. We note that

$$
B(z, w)^{-1}=(1-\langle z, w\rangle)^{-2}\left((1-\langle z, w\rangle) I+z \otimes w^{*}\right) .
$$

Let $B^{t}(z, w)$ denote the dual of $B(z, w)$ acting on the dual space $V^{\prime}$ of $V$. When acting on a vector $v^{\prime} \in V^{\prime}$ it is

$$
B^{t}(z, w) v^{\prime}=(1-\langle z, w\rangle) v^{\prime}\left(I-z \bar{w}^{t}\right) .
$$

For a nonnegative integer $s$, let $\otimes^{s} V^{\prime}$ be the tensor product of $s$ copies of $V^{\prime}$ and let $\otimes^{0} V^{\prime}=\mathbb{C}$. The space $\otimes^{s} V^{\prime}$ is equipped with a natural Hermitian inner product induced by that of $V^{\prime}$. Denote by $\odot^{s} V^{\prime}$ the subspace of symmetric tensors of length $s$ and denote by $\otimes^{s} B^{t}(z, z)$ the operator on $\otimes^{s} V^{\prime}$ induced by the action of $B^{t}(z, z)$ on $V^{\prime}$, where $\otimes^{0} B^{t}(z, z)=I$. Recall, generally, that if $A$ acts on $V^{\prime}$, $\otimes^{s} A$ acts on $\otimes^{s} V^{\prime}$ by

$$
\left(\otimes^{s} A\right)\left(u_{1} \otimes u_{2} \otimes \cdots \otimes u_{s}\right)=\left(A u_{1}\right) \otimes\left(A u_{2}\right) \otimes \cdots \otimes\left(A u_{s}\right) .
$$

For example, in the case $s=2$ the operator $\otimes^{2} B^{t}(z, z)$ becomes

$$
\left(1-|z|^{2}\right)^{2}\left(I \otimes I-I \otimes A_{z}-A_{z} \otimes I+A_{z} \otimes A_{z}\right),
$$

where $A_{z}=\bar{z} \otimes \bar{z}^{*}$. Let $L_{\nu, s}^{p}=L_{\nu}^{p}\left(\mathbb{B}, \odot{ }^{s} V^{\prime}\right)$ be the space of functions $G: \mathbb{B} \rightarrow \odot^{s} V^{\prime}$ such that

$$
\|G\|_{\nu, s, p}=\left(\int_{\mathbb{B}}\left\langle\left(1-|z|^{2}\right)^{2 \nu} \otimes^{s} B^{t}(z, z) G(z), G(z)\right\rangle^{p / 2} d \iota(z)\right)^{1 / p}<\infty,
$$

where $1 \leq p<\infty$, and let $L_{\nu, s}^{\infty}$ be the space of functions $G: \mathbb{B} \rightarrow \odot{ }^{s} V^{\prime}$ such that

$$
\|G\|_{\nu, s, \infty}=\sup _{z \in \mathbb{B}}\left\langle\left(1-|z|^{2}\right)^{2 \nu} \otimes^{s} B^{t}(z, z) G(z), G(z)\right\rangle^{1 / 2}<\infty .
$$

Let $\mathcal{H}_{\nu, s}^{p}$ be the closed subspace of all holomorphic functions in $L_{\nu, s}^{p}, 1 \leq p \leq \infty$. 
Also, we need the group $G$ of biholomorphic mappings of $\mathbb{B}$. Let $P_{z}$ be the orthogonal projection of $\mathbb{C}^{d}$ onto $\mathbb{C} z$ and let $Q_{z}=I-P_{z}$. Put $s_{z}=\left(1-|z|^{2}\right)^{1 / 2}$ and define a linear fractional mapping $\varphi_{z}$ on $\mathbb{B}$ by (see $[\underline{\mathrm{Ru}}]$ )

$$
\varphi_{z}(w)=\frac{z-P_{z} w-s_{z} Q_{z} w}{1-\langle w, z\rangle} .
$$

If $g \in G$ and $g(z)=0$, then there is a unique unitary operator $U: \mathbb{C}^{d} \rightarrow \mathbb{C}^{d}$ such that

$$
g=U \varphi_{z}
$$

Define the complex Jacobian $J_{g}$ by $J_{g}(w)=\operatorname{det}\left(g^{\prime}(w)\right)$. Now, let $z_{0} \in \mathbb{B}$. Then by arguments in Remark $3.1 \mathrm{in}[\mathrm{Su}$ it follows that there is a constant $c$ with $|c|=1$ such that

$$
J_{\varphi_{z_{0}}}(w)^{2 \nu /(d+1)}=c \cdot \frac{\left(1-\left|z_{0}\right|^{2}\right)^{\nu}}{\left(1-\left\langle w, z_{0}\right\rangle\right)^{2 \nu}} .
$$

The next theorem gives the reproducing properties for $\mathcal{H}_{\nu, s}^{p}$.

Theorem 2.1. Let $1 \leq p \leq \infty$. There is a nonzero constant $c$ such that, for any $G \in \mathcal{H}_{\nu, s}^{p}$ and any $v \in \odot^{s} V^{\prime}$,

$$
\langle G(z), v\rangle=c \int_{\mathbb{B}}\left\langle\otimes^{s} B^{t}(w, w) G(w), K_{\nu, s}(w, z) v\right\rangle\left(1-|w|^{2}\right)^{2 \nu} d \iota(w),
$$

where

$$
K_{\nu, s}(w, z)=(1-\langle w, z\rangle)^{-2 \nu} \otimes^{s} B^{t}(w, z)^{-1} .
$$

The proof of this theorem is given at the end of this subsection.

Remark 2.2. Consider $\mathcal{H}_{\nu, s}^{2} \subset L_{\nu, s}^{2}$. According to Lemma $3.5 \mathrm{in}[\mathrm{Su}$ ] the orthogonal projection operator $P_{\nu, s}$ of $L_{\nu, s}^{2}$ onto $\mathcal{H}_{\nu, s}^{2}$, is given by

$$
P_{\nu, s} G(z)=c \int_{\mathbb{B}}\left(1-|w|^{2}\right)^{2 \nu} K_{\nu, s}(z, w) \otimes^{s} B^{t}(w, w) G(w) d \iota(w) .
$$

Namely, for any $G \in L_{\nu, s}^{2}$ and any $v \in \odot^{s} V^{\prime}$ it follows that

$$
\left\langle P_{\nu, s} G(z), v\right\rangle=c \int_{\mathbb{B}}\left\langle\otimes^{s} B^{t}(w, w) G(w), K_{\nu, s}(w, z) v\right\rangle\left(1-|w|^{2}\right)^{2 \nu} d \iota(w) .
$$

The orthogonal projection operator has the following boundedness property.

Proposition 2.3. If $1 \leq p<\infty$, then $P_{\nu, s}: L_{\nu, s}^{p} \rightarrow \mathcal{H}_{\nu, s}^{p}$ is bounded.

Proof. The case $1<p<\infty$ is just Corollary $7.4 \mathrm{in}[\mathrm{Su}$. Now, consider the case $p=1$. Let $F \in L_{\nu, s}^{1}$. Then it follows from Theorem 2.1 above and Lemma 7.1 in $\mathrm{Su}$ ] that

$$
\left\|\otimes^{s} B^{t}(z, z)^{1 / 2} P_{\nu, s} F(z)\right\| \leq C_{s} \int_{\mathbb{B}} T(z, w)\left\|\otimes^{s} B^{t}(w, w)^{1 / 2} F(w)\right\|\left(1-|w|^{2}\right)^{2 \nu} d \iota(w),
$$

where

$$
T(z, w)=\frac{\left(1-|z|^{2}\right)^{s / 2}\left(1-|w|^{2}\right)^{s / 2}}{|1-\langle z, w\rangle|^{2 \nu+s}}
$$


Thus, by Fubini-Tonelli's theorem and Proposition 1.4.10 in [Ru it follows that

$$
\begin{aligned}
\left\|P_{\nu, s} F\right\|_{\nu, s, 1} \leq & C_{s} \int_{\mathbb{B}}\left\|\otimes^{s} B^{t}(w, w)^{1 / 2} F(w)\right\|\left(1-|w|^{2}\right)^{2 \nu} \\
& \cdot\left(\int_{\mathbb{B}} T(z, w)\left(1-|z|^{2}\right)^{\nu} d \iota(z)\right) d \iota(w) \\
\leq & C_{s}^{\prime} \int_{\mathbb{B}}\left\|\otimes^{s} B^{t}(w, w)^{1 / 2} F(w)\right\|\left(1-|w|^{2}\right)^{\nu} d \iota(w)=C_{s}^{\prime}\|F\|_{\nu, s, 1} .
\end{aligned}
$$

Note that it is proved in $\mathrm{Su}$, using the complex interpolation method of Banach spaces, that $\mathcal{H}_{\nu, s}^{p}=\left(\mathcal{H}_{\nu, s}^{2}, \mathcal{H}_{\nu, s}^{\infty}\right)_{[1-2 / p]}$ if $2<p<\infty$; see Theorem 8.2 in [Su]. However, Proposition 2.3 allows us to use the same proof as in $\mathrm{Su}$ to get the following result.

Corollary 2.4. If $1<p<\infty$, then

$$
\mathcal{H}_{\nu, s}^{p}=\left(\mathcal{H}_{\nu, s}^{1}, \mathcal{H}_{\nu, s}^{\infty}\right)_{[1-1 / p]} .
$$

Now we go back to Theorem 2.1. First we need a proposition.

Proposition 2.5. Let $s$ be a nonnegative integer and let $\nu>d, 2 \nu>\alpha>d$. Then there is a constant $C_{s}>0$ such that

$$
\left(1-|z|^{2}\right)^{2 \nu-\alpha}\left\|K_{\nu, s}(\cdot, z) \otimes^{s} B^{t}(z, z)^{1 / 2} v\right\|_{\alpha, s, 1} \leq C_{s}\|v\|
$$

for all $z \in \mathbb{B}$ and all $v \in \odot^{s} V^{\prime}$.

Proof. Let $v \in \odot{ }^{s} V^{\prime}$. It follows from Lemma $7.1 \mathrm{in}[\mathrm{Su}$ and Proposition 1.4.10 in $[\mathrm{Ru}$ that

$$
\begin{aligned}
& \left\|K_{\nu, s}(\cdot, z) \otimes^{s} B^{t}(z, z)^{1 / 2} v\right\|_{\alpha, s, 1} \\
& \quad=\int_{\mathbb{B}}\left\|\otimes^{s} B^{t}(w, w)^{1 / 2} \otimes^{s} B^{t}(w, z)^{-1} \otimes^{s} B^{t}(z, z)^{1 / 2} v\right\| \frac{\left(1-|w|^{2}\right)^{\alpha}}{|1-\langle w, z\rangle|^{2 \nu}} d \iota(w) \\
& \quad \leq \quad C_{s}\|v\| \int_{\mathbb{B}} \frac{\left(1-|z|^{2}\right)^{s / 2}\left(1-|w|^{2}\right)^{\alpha+s / 2}}{|1-\langle w, z\rangle|^{2 \nu+s}} d \iota(w) \leq C_{s}^{\prime}\left(1-|z|^{2}\right)^{\alpha-2 \nu}\|v\| .
\end{aligned}
$$

Lemma 2.6. Let $z \in \mathbb{B}$. Then there is a constant $C_{s}>0$ such that, for any $v \in \odot{ }^{s} V^{\prime}$ and any $1 \leq p \leq \infty$, it follows that

$$
\left\|\left(1-|z|^{2}\right)^{\nu} K_{\nu, s}(\cdot, z) \otimes^{s} B^{t}(z, z)^{1 / 2} v\right\|_{\nu, s, p} \leq C_{s}\|v\| .
$$

Proof. Let $T_{z}=\left(1-|z|^{2}\right)^{\nu} K_{\nu, s}(\cdot, z) \otimes^{s} B^{t}(z, z)^{1 / 2}$. By Proposition 2.5 and by Lemma 7.1 in $\left[\mathrm{Su}\right.$ it follows that $\left\|T_{z} v\right\|_{\nu, s, 1} \leq C_{s}\|v\|$ and $\left\|T_{z} v\right\|_{\nu, s, \infty} \leq C_{s}^{\prime}\|v\|$ respectively, for all $v \in \odot{ }^{s} V^{\prime}$. Thus the result follows from Riesz-Thorin's interpolation theorem.

Now we can prove Theorem 2.1 .

Proof of Theorem 2.1. Let $G \in \mathcal{H}_{\nu, s}^{p}, 1 \leq p \leq \infty$. Then it follows from Lemma 2.6 that, for all $v \in \odot{ }^{s} V^{\prime}$,

$$
\int_{\mathbb{B}}\left|\left\langle\otimes^{s} B^{t}(w, w) G(w), K_{\nu, s}(w, z) v\right\rangle\right| d \iota_{2 \nu}(w) \leq\|G\|_{\nu, s, p}\left\|K_{\nu, s}(\cdot, z) v\right\|_{\nu, s, q}<\infty .
$$


In particular, if $z=0$, then

$$
\int_{\mathbb{B}}\left|\left\langle\otimes^{s} B^{t}(w, w) G(w), v\right\rangle\right|\left(1-|w|^{2}\right)^{2 \nu} d \iota(w)<\infty .
$$

By the mean-value property for holomorphic functions and rotation invariance for integration,

$$
\int_{\mathbb{B}}\left\langle\left(1-|w|^{2}\right)^{2 \nu} \otimes^{s} B^{t}(w, w) G(w), v\right\rangle d \iota(w)=c^{\prime}\langle G(0), v\rangle,
$$

where $c^{\prime} \neq 0$ only depends on $d, \nu$ and $s$. Hence, there exists a nonzero constant $c$ such that, for all $G \in \mathcal{H}_{\nu, s}^{p}$ and all $v \in \odot{ }^{s} V^{\prime}$,

$$
\langle G(0), v\rangle=c\langle G, v\rangle_{\nu, s, 2},
$$

where $\langle\cdot, \cdot\rangle_{\nu, s, 2}$ is the $\mathcal{H}_{\nu, s}^{2}$-pairing. Now, define an isometry $\pi_{\nu, s}$ on $\mathcal{H}_{\nu, s}^{2}$ by

$$
\pi_{\nu, s}: g \in G, S(z) \rightarrow\left(\otimes^{s}\left(d g^{-1}(z)\right)^{t}\right) S\left(g^{-1} z\right)\left(J_{g^{-1}}(z)\right)^{2 \nu /(d+1)},
$$

as in $\left[\mathrm{Su}\right.$. Let $z_{0} \in \mathbb{B}$. For notational convenience we prove the reproducing property only for $s=1$; the case for general $s$ is identically the same. On the one hand,

$$
\left\langle\left(\pi_{\nu, 1}\left(\varphi_{z_{0}}\right) G\right)(0), v\right\rangle=\left\langle G\left(z_{0}\right),{\overline{J_{\varphi_{0}}}}{ }^{2 \nu}{ }^{2 \nu(d+1)}\left(\varphi_{z_{0}}^{\prime}(0)^{t}\right)^{*} v\right\rangle .
$$

By equation (2.6),$\left(1-\left|z_{0}\right|^{2}\right)^{(d+1) / 2}<\left|J_{\varphi_{z_{0}}}(w)\right|<\left(1-\left|z_{0}\right|^{2}\right)^{-d-1}$ on $\mathbb{B}$, so $\pi_{\nu, 1}\left(\varphi_{z_{0}}\right) G \in \mathcal{H}_{\nu, 1}^{p}$. However, using equation (2.8) above for $\pi_{\nu, 1}\left(\varphi_{z_{0}}\right) G$ and the transformation properties

$$
B\left(\varphi_{z_{0}}(w), \varphi_{z_{0}}(z)\right)=\varphi_{z_{0}}^{\prime}(w) B(w, z)\left(\varphi_{z_{0}}^{\prime}(z)\right)^{*}
$$

(see equation (9) in $[\underline{\mathrm{Su}}$ ) and

$$
\begin{aligned}
K_{\nu, 1}\left(\varphi_{z_{0}}(w), \varphi_{z_{0}}(z)\right)= & J_{\varphi_{z_{0}}}(w)^{-2 \nu /(d+1)} \cdot{\overline{J_{\varphi_{0}}(z)}}^{-2 \nu /(d+1)} \\
& \cdot\left(\varphi_{z_{0}}^{\prime}(w)^{t}\right)^{-1} K_{\nu, 1}(w, z)\left(\overline{\varphi_{z_{0}}^{\prime}(z)}\right)^{-1}
\end{aligned}
$$

(see equation (9) in $[\mathrm{Su}$, and Theorem 2.2.5 in $[\mathrm{Ru}]$ ), the left-hand side in equation (2.9) above is

$$
\left\langle G\left(z_{0}\right), u\right\rangle=c\left\langle G, K_{\nu, s}\left(\cdot, z_{0}\right) u\right\rangle_{\nu, s, 2},
$$

where $u={\overline{J_{\varphi_{0}}}}^{2 \nu}{ }^{2 \nu /(d+1)}\left(\varphi_{z_{0}}^{\prime}(0)^{t}\right)^{*} v$. Since $v$ is arbitrary, then so is $u \in \odot^{s} V^{\prime}$, which proves the theorem.

2.2. Hankel forms of higher weights. Let $H_{1}$ and $H_{2}$ be Hilbert spaces and let $T: H_{1} \rightarrow H_{2}$ be a linear operator. Define the singular numbers $s_{n}(T)=$ $\inf \{\|T-K\|: \operatorname{rank}(K) \leq n\}, n \geq 0$. If $T$ is compact, these singular numbers are equal to the eigenvalues of $|T|=\left(T^{*} T\right)^{1 / 2}$. We denote by $\mathcal{S}_{p}$ the ideal of operators for which $\left\{s_{n}(T)\right\}_{n \geq 0} \in l^{p}, 0<p \leq \infty$; see [S].

The transvectant $\mathcal{T}_{s}$ on $L_{a}^{2}\left(d \iota_{\nu}\right) \otimes L_{a}^{2}\left(d \iota_{\nu}\right)$ (introduced in [P1]; see also [P2], [PZ] and $[\underline{\mathrm{Su}}]$ ) is defined by

$$
\mathcal{T}_{s}(f, g)(z)=\sum_{k=0}^{s}\left(\begin{array}{l}
s \\
k
\end{array}\right)(-1)^{k} \frac{\partial^{s-k} f(z) \odot \partial^{k} g(z)}{(\nu)_{s-k}(\nu)_{k}},
$$


where

$$
\partial^{s} f(z)=\sum_{j_{1} \ldots j_{s}=1}^{d} \partial_{j_{1}} \cdots \partial_{j_{s}} f(z) d z_{j_{1}} \otimes \cdots \otimes d z_{j_{s}} \in \odot^{s} V^{\prime}
$$

and $(\nu)_{k}=\nu(\nu+1) \cdots(\nu+k-1),(\nu)_{0}=1$, is the Pochammer symbol.

Lemma 2.7. There is a constant $C_{s}>0$ such that

$$
\left\|\mathcal{T}_{s}(f, g)\right\|_{\nu, s, 1} \leq C_{s}\|f\|_{\nu}\|g\|_{\nu}
$$

for all $f, g \in L_{a}^{2}\left(d \iota_{\nu}\right)$.

First we need a lemma, which actually is a consequence of Theorem $4.1 \mathrm{in} \underline{\mathrm{Su}}$, but we give an independent and easier proof.

Lemma 2.8. There is a constant $C_{\nu, s}>0$ such that

$$
\int_{\mathbb{B}}\left\langle\otimes^{s} B^{t}(z, z) \partial^{s} f(z), \partial^{s} f(z)\right\rangle\left(1-|z|^{2}\right)^{\nu} d \iota(z) \leq C_{\nu, s}\|f\|_{\nu}
$$

for all $f \in L_{a}^{2}\left(d \iota_{\nu}\right)$.

Proof. First,

$$
\partial^{s} f(z)=c_{\nu}(\nu)_{s} \int_{\mathbb{B}} \frac{f(w) \otimes^{s} \bar{w}}{(1-\langle z, w\rangle)^{\nu+s}} \cdot\left(1-|w|^{2}\right)^{\nu} d \iota(w),
$$

so that

$$
\left\|\otimes^{s} B^{t}(z, z)^{1 / 2} \partial^{s} f(z)\right\| \leq C_{\nu, s} \int_{\mathbb{B}} \frac{|f(w)| \cdot\left\|B^{t}(z, z)^{1 / 2} \bar{w}\right\|^{s}}{|1-\langle z, w\rangle|^{\nu+s}} \cdot\left(1-|w|^{2}\right)^{\nu} d \iota(w) .
$$

We can estimate

$$
\begin{aligned}
\left\|B^{t}(z, z)^{1 / 2} \bar{w}\right\| & =s_{z}\left(\left\|s_{z} P_{\bar{z}} \bar{w}\right\|^{2}+\left\|Q_{\bar{z}} \bar{w}\right\|^{2}\right)^{1 / 2} \\
& =s_{z}\left(|w|^{2}-|\langle z, w\rangle|^{2}\right)^{1 / 2} \\
& \leq \sqrt{2} \cdot s_{z}|1-\langle z, w\rangle|^{1 / 2} .
\end{aligned}
$$

Hence,

$$
\left\|\otimes^{s} B^{t}(z, z)^{1 / 2} \partial^{s} f(z)\right\| \leq C_{\nu, s}^{\prime} \int_{\mathbb{B}} T(z, w)|f(w)|\left(1-|w|^{2}\right)^{\nu} d \iota(w),
$$

where

$$
T(z, w)=\frac{\left(1-|z|^{2}\right)^{s / 2}}{|1-\langle z, w\rangle|^{\nu+s / 2}} .
$$

Now, the result follows by exactly the same arguments as in the proof of Theorem 7.2 in $[\mathrm{Su}]$ (where we let $t=-(\nu-d) / 4$ ).

Proof of Lemma 2.7. The transvectant is a linear combination of terms $\partial^{k} f(z) \otimes$ $\partial^{s-k} g(z)$ so we need only to estimate $\left\|\partial^{k} f(z) \otimes \partial^{s-k} g(z)\right\|_{\nu, s, 1}$ for $0 \leq k \leq s$. First we observe that

$$
\begin{aligned}
& \left\|\otimes^{s} B^{t}(z, z)^{1 / 2} \partial^{k} f(z) \otimes \partial^{s-k} g(z)\right\| \\
& \quad=\left\|\otimes^{k} B^{t}(z, z)^{1 / 2} \partial^{k} f(z)\right\| \cdot\left\|\otimes^{s-k} B^{t}(z, z)^{1 / 2} \partial^{s-k} g(z)\right\| .
\end{aligned}
$$


Thus by Hölder's inequality and Lemma 2.8 it follows that

$$
\begin{aligned}
& \int_{\mathbb{B}} \| \otimes^{s} B^{t}(z, z)^{1 / 2} \partial^{k} f(z) \otimes \partial^{s-k} g(z) \|\left(1-|z|^{2}\right)^{\nu} d \iota(z) \\
& \leq C\|f\|_{\nu, k}\|g\|_{\nu, s-k} \leq C_{s}\|f\|_{\nu}\|g\|_{\nu} .
\end{aligned}
$$

The Hankel bilinear form $H_{F}^{s}$ on $L_{a}^{2}\left(d \iota_{\nu}\right) \otimes L_{a}^{2}\left(d \iota_{\nu}\right)$ is defined by

$$
H_{F}^{s}(f, g)=\int_{\mathbb{B}}\left\langle\otimes^{s} B^{t}(z, z) \mathcal{T}_{s}(f, g)(z), F(z)\right\rangle d \iota_{2 \nu}(z)
$$

where $F: \mathbb{B} \rightarrow \odot^{s} V^{\prime}$ is holomorphic. We call $F$ the symbol of the corresponding Hankel form. We remark that

$$
H_{F}^{0}(f, g)=\int_{\mathbb{B}} f(z) g(z) \overline{F(z)} d \iota_{2 \nu}(z) .
$$

This is the classical Hankel form studied in [JPR].

With the form $H_{F}^{s}$ one can associate the operator $A_{F}^{s}$ defined by

$$
H_{F}^{s}(f, g)=\left\langle f, A_{F}^{s} g\right\rangle_{\nu}
$$

as in [JPR]. Notice that $A_{F}^{s}$ is an anti-linear operator on $L_{a}^{2}\left(d \iota_{\nu}\right)$. To get a linear operator one combines $A_{F}^{s}$ with a conjugation, i.e., one instead considers the operator $\bar{A}_{F}^{s}: g \rightarrow \overline{A_{F}^{s} g}$. We say that $H_{F}^{s}$ is of Schatten-von Neumann class $\mathcal{S}_{p}$, for $0<p<\infty$, if and only if $\bar{A}_{F}^{s}: L_{a}^{2}\left(d \iota_{\nu}\right) \rightarrow \overline{L^{2}\left(d \iota_{\nu}\right)}$ is of class $\mathcal{S}_{p}$.

\section{Duality of $\mathcal{H}_{\nu, s}^{p}$}

In this section we determine the dual space $\left(\mathcal{H}_{\nu, s}^{p}\right)^{*}$ of $\mathcal{H}_{\nu, s}^{p}, 1 \leq p<\infty$.

Lemma 3.1. Let $1 \leq p<\infty$. If $\Phi \in\left(L_{\nu, s}^{p}\right)^{*}$, then there is a function $G \in L_{\nu, s}^{q}$ such that

$$
\Phi(F)=\int_{\mathbb{B}}\left\langle\otimes^{s} B^{t}(z, z) F(z), G(z)\right\rangle\left(1-|z|^{2}\right)^{2 \nu} d \iota(z)
$$

and $\|\Phi\|=\|G\|_{\nu, s, q}$ where $1 / q+1 / p=1$.

Proof. Define $A(z)=\left(1-|z|^{2}\right)^{\nu} \otimes^{s} B^{t}(z, z)^{1 / 2}$ and $\left(M_{A} F\right)(z)=A(z) F(z)$. Then $M_{A}$ is an isometry from $L_{\nu, s}^{p}$ onto $L^{p}$, where $L^{p}=\left\{F: \mathbb{B} \rightarrow V:\|F\|_{p}<\infty\right\}$ and

$$
\|F\|_{p}=\left(\int_{\mathbb{B}}\|F(z)\|^{p} d \iota(z)\right)^{1 / p} .
$$

Consider $\Theta=\Phi M_{A}^{-1}$. Then $\Theta$ is a bounded linear functional on $L^{p}$ and $\Theta(A F)=$ $\Phi(F)$. Then we can find a function $H \in L^{q}$ such that

$$
\Phi(F)=\int_{\mathbb{B}}\langle(A F)(z), H(z)\rangle d \iota(z)
$$

with $\|\Theta\|=\|H\|_{q}$. Let $G=M_{A}^{-1} H$. Then $G \in L_{\nu, s}^{q}$ and

$$
\Phi(F)=\int_{\mathbb{B}}\left\langle\otimes^{s} B^{t}(z, z) F(z), G(z)\right\rangle\left(1-|z|^{2}\right)^{2 \nu} d \iota(z) .
$$

Also $\|\Phi\|=\|G\|_{\nu, s, q}$. 
Theorem 3.2. For $1 \leq p<\infty$ we have $\left(\mathcal{H}_{\nu, s}^{p}\right)^{*}=\mathcal{H}_{\nu, s}^{q}$, under the integral pairing

$$
\langle F, G\rangle_{\nu, s, 2}=\int_{\mathbb{B}}\left\langle\otimes^{s} B^{t}(z, z) F(z), G(z)\right\rangle\left(1-|z|^{2}\right)^{2 \nu} d \iota(z), \quad F \in \mathcal{H}_{\nu, s}^{p}, G \in \mathcal{H}_{\nu, s}^{q},
$$

where $1 / p+1 / q=1$. Namely, for any bounded linear functional $\Phi: \mathcal{H}_{\nu, s}^{p} \rightarrow \mathbb{C}$ there is a function $G \in \mathcal{H}_{\nu, s}^{q}$ such that $\Phi(F)=\langle F, G\rangle_{\nu, s, 2}$ for all $F \in \mathcal{H}_{\nu, s}^{p}$ with

$$
C\|G\|_{\nu, s, q} \leq\|\Phi\| \leq\|G\|_{\nu, s, q} .
$$

Proof. By Hölder's inequality, every function $G \in \mathcal{H}_{\nu, s}^{q}$ defines a bounded linear functional $\Phi$ on $\mathcal{H}_{\nu, s}^{p}$ under the above integral pairing with $\|\Phi\| \leq\|G\|_{\nu, s, q}$.

Conversely, let $\Phi \in\left(\mathcal{H}_{\nu, s}^{p}\right)^{*}$. By the Hahn-Banach theorem we can extend $\Phi$ to a bounded linear functional $\tilde{\Phi}$ on $L_{\nu, s}^{p}$ such that $\Phi(F)=\tilde{\Phi}(F)$ for all $F \in \mathcal{H}_{\nu, s}^{p}$ with $\|\Phi\|=\|\tilde{\Phi}\|$. By Lemma 3.1 there is a function $H \in L_{\nu, s}^{q}$ such that

$$
\tilde{\Phi}(F)=\int_{\mathbb{B}}\left\langle\otimes^{s} B^{t}(z, z) F(z), H(z)\right\rangle\left(1-|z|^{2}\right)^{2 \nu} d \iota(z)
$$

for all $F \in L_{\nu, s}^{p}$, with $\|\tilde{\Phi}\|=\|H\|_{\nu, s, q}$. However, Theorem 2.1 implies that, for any $F \in \mathcal{H}_{\nu, s}^{p}$,

$$
F(z)=\left(P_{\nu, s} F\right)(z)=c \int_{\mathbb{B}}\left(1-|w|^{2}\right)^{2 \nu} K_{\nu, s}(w, z)^{*} \otimes^{s} B^{t}(w, w) F(w) d \iota(w) .
$$

Substituting this into formula (3.1) and using Fubini-Tonelli's theorem we get that

$$
\Phi(F)=\tilde{\Phi}(F)=\int_{\mathbb{B}}\left\langle\otimes^{s} B^{t}(w, w) F(w),\left(P_{\nu, s} H\right)(w)\right\rangle\left(1-|w|^{2}\right)^{2 \nu} d \iota(w) .
$$

Let $G=P_{\nu, s} H$. By Proposition 2.3, $\left\|P_{\nu, s} H\right\|_{\nu, s, q} \leq C^{\prime}\|H\|_{\nu, s, q}$. Then $G \in \mathcal{H}_{\nu, s}^{q}$, $\Phi(F)=\langle F, G\rangle_{\nu, s, 2}$ for all $F \in \mathcal{H}_{\nu, s}^{p}$ and $C\|G\|_{\nu, s, q} \leq\|\Phi\|$.

\section{4. Аtomic Decomposition of $\mathcal{H}_{\nu, s}^{1}$}

Following [JPR, we denote by $l^{1}\left(\mathbb{B}, \odot^{s} V^{\prime}\right)$ the space of all functions $a: \mathbb{B} \rightarrow$ $\odot^{s} V^{\prime}$, with support in $\left\{z_{j}\right\}_{j=1}^{\infty} \subset \mathbb{B}$, such that

$$
\|a\|_{l^{1}}=\sum_{j=1}^{\infty}\left\|a\left(z_{j}\right)\right\|<\infty .
$$

Also, denote by $l^{\infty}\left(\mathbb{B}, \odot^{s} V^{\prime}\right)$ the space of all functions $a: \mathbb{B} \rightarrow \odot{ }^{s} V^{\prime}$ such that

$$
\|a\|_{l \infty}=\sup _{z \in \mathbb{B}}\|a(z)\|<\infty .
$$

Then it is elementary that

$$
l^{\infty}\left(\mathbb{B}, \odot^{s} V^{\prime}\right)=\left(l^{1}\left(\mathbb{B}, \odot^{s} V^{\prime}\right)\right)^{*},
$$

under the pairing

$$
\langle a, b\rangle^{\prime}=\sum_{j=1}^{\infty}\left\langle a\left(z_{j}\right), b\left(z_{j}\right)\right\rangle
$$

where $a \in l^{1}\left(\mathbb{B}, \odot^{s} V^{\prime}\right)$ with support $\left\{z_{j}\right\}_{j=1}^{\infty} \subset \mathbb{B}$ and $b \in l^{\infty}\left(\mathbb{B}, \odot^{s} V^{\prime}\right)$. Namely, for any bounded linear functional $\Phi: l^{1}\left(\mathbb{B}, \odot^{s} V^{\prime}\right) \rightarrow \mathbb{C}$ there is a function $b$ in $l^{\infty}\left(\mathbb{B}, \odot^{s} V^{\prime}\right)$ such that $\Phi(a)=\langle a, b\rangle^{\prime}$ for all $a \in l^{1}\left(\mathbb{B}, \odot^{s} V^{\prime}\right)$ with $\|\Phi\|=\|b\|_{l^{\infty}}$. 
Theorem 4.1. It follows that $F \in \mathcal{H}_{\nu, s}^{1}$ if and only if there is a sequence $\left\{z_{j}\right\}_{j=1}^{\infty} \subset$ $\mathbb{B}$ and a sequence $\left\{a_{j}\right\}_{j=1}^{\infty} \in l^{1}\left(\mathbb{B}, \odot{ }^{s} V^{\prime}\right)$ such that

$$
F(w)=\sum_{j=1}^{\infty}\left(1-\left|z_{j}\right|^{2}\right)^{\nu} K_{\nu, s}\left(w, z_{j}\right) \otimes^{s} B^{t}\left(z_{j}, z_{j}\right)^{1 / 2} a_{j} .
$$

Proof. By Proposition 2.5, for any $v \in \odot^{s} V^{\prime}$ and any $z \in \mathbb{B}$,

$$
\left\|K_{\nu, s}(\cdot, z) \otimes^{s} B^{t}(z, z)^{1 / 2} v\right\|_{\nu, s, 1} \leq C_{s}\left(1-|z|^{2}\right)^{-\nu}\|v\| .
$$

Thus, the operator $T: l^{1}\left(\mathbb{B}, \odot{ }^{s} V^{\prime}\right) \rightarrow \mathcal{H}_{\nu, s}^{1}$ defined by

$$
(T a)(w)=\sum_{j=1}^{\infty}\left(1-\left|z_{j}\right|^{2}\right)^{\nu} K_{\nu, s}\left(w, z_{j}\right) \otimes^{s} B^{t}\left(z_{j}, z_{j}\right)^{1 / 2} a_{j}
$$

is bounded, where $a_{j}=a\left(z_{j}\right)$ and the support of $a$ is $\left\{z_{j}\right\}_{j=1}^{\infty}$. We need to prove that $T$ is onto. Consider $T^{*}:\left(\mathcal{H}_{\nu, s}^{1}\right)^{*} \rightarrow\left(l^{1}\left(\mathbb{B}, \odot^{s} V^{\prime}\right)\right)^{*}, T^{*}(\Phi)(a)=\Phi(T a)$, which is bounded, where $\Phi \in\left(\mathcal{H}_{\nu, s}^{1}\right)^{*}$ and $a \in l^{1}\left(\mathbb{B}, \odot^{s} V^{\prime}\right)$. By Theorem 3.2, for any $\Phi \in\left(\mathcal{H}_{\nu, s}^{1}\right)^{*}$ there is a $G \in \mathcal{H}_{\nu, s}^{\infty}$ such that $\Phi(F)=\langle F, G\rangle_{\nu, s, 2}$ for all $F \in \mathcal{H}_{\nu, s}^{1}$ with $C\|G\|_{\nu, s, \infty} \leq\|\Phi\| \leq\|G\|_{\nu, s, \infty}$. Now, let $a \in l^{1}\left(\mathbb{B}, \odot{ }^{s} V^{\prime}\right)$ with support $\left\{z_{j}\right\}_{j=1}^{\infty} \subset \mathbb{B}$. By the reproducing property in Theorem 2.1 it follows that

$$
T^{*}(\Phi)(a)=\Phi(T a)=\langle T a, G\rangle_{\nu, s, 2}=c \sum_{j=1}^{\infty}\left\langle a_{j},\left(1-\left|z_{j}\right|^{2}\right)^{\nu} \otimes B^{t}\left(z_{j}, z_{j}\right) G\left(z_{j}\right)\right\rangle .
$$

Hence, by (4.1) and Theorem 3.2 it follows that

$$
\frac{1}{c} \cdot\left\|T^{*} \Phi\right\|_{\left(l^{1}\right)^{*}}=\sup _{z \in \mathbb{B}}\left\|\left(1-|z|^{2}\right)^{\nu} \otimes^{s} B^{t}(z, z) G(z)\right\|=\|G\|_{\nu, s, \infty} \geq\|\Phi\| .
$$

On the one hand, (4.2) yields that $\operatorname{ker} T^{*}=\{0\}$ and consequently the range of $T$ is dense in $\mathcal{H}_{\nu, s}^{1}$. On the other hand, (4.2) yields that the range of $T^{*}$ is closed and so is the range of $T$ by the Closed Range Theorem.

\section{Trace Class $\mathcal{S}_{1}$}

We consider now the trace class property of $H_{F}^{s}$ in (2.11).

Theorem 5.1. The Hankel form $H_{F}^{s}$ is of trace class $\mathcal{S}_{1}$ if and only if $F \in \mathcal{H}_{\nu, s}^{1}$.

Combining the results in $\mathrm{Su}$ ] we have now a complete characterization of the Schatten-von Neumann class Hankel forms.

Theorem 5.2. The Hankel form $H_{F}^{s}$ is of Schatten-von Neumann class $\mathcal{S}_{p}$ if and only if $F \in \mathcal{H}_{\nu, s}^{p}, 1 \leq p \leq \infty$.

Proof of Theorem 5.2. It follows from Lemma 5.5below and Theorem 1.1(a) in [Su] that the operator $\Gamma: F \rightarrow H_{F}^{s}$ is bounded from $\mathcal{H}_{\nu, s}^{1}$ into $\mathcal{S}_{1}$ and from $\mathcal{H}_{\nu, s}^{\infty}$ into $\mathcal{S}_{\infty}$, respectively. Since $\mathcal{S}_{p}=\left(\mathcal{S}_{1}, \mathcal{S}_{\infty}\right)_{[1-1 / p]}$ if $1<p<\infty$, then it follows by RieszThorin's interpolation theorem and Corollary 2.4 that $\Gamma$ is bounded from $\mathcal{H}_{\nu, s}^{p}$ into $\mathcal{S}_{p}$ if $1<p<\infty$.

On the other hand, it follows from Lemma 5.6 below and Theorem 1.1(a) in $\mathrm{Su}$ that $\tilde{\mathcal{T}}_{s}$, defined in (5.2), is bounded from $\mathcal{S}_{1}$ into $\mathcal{H}_{\nu, s}^{1}$ and from $\mathcal{S}_{\infty}$ into $\mathcal{H}_{\nu, s}^{\infty}$, respectively. Again, by interpolation $\tilde{\mathcal{T}}_{s}$ is bounded from $\mathcal{S}_{p}$ into $\mathcal{H}_{\nu, s}^{p}$ if $1<p<\infty$. 
Also, if $H_{F}^{s} \in \mathcal{S}_{p}$ for $1 \leq p<\infty$, then $\tilde{\mathcal{T}}_{s}\left(H_{F}^{s}\right)=F$, which follows by the same arguments as in the proof of Lemma 8.6 in $[\mathrm{Su}]$.

The proof of Theorem 5.1 will be divided into a few lemmas. We will first show in Lemma 5.3 that every $H_{F}^{s}$ is of trace class $\mathcal{S}_{1}$ if $F$ is in $\mathcal{H}_{\nu, s}^{1}$ and then in Lemma 5.4 that $\mathcal{H}_{\nu, s}^{1}$ can be continuously embedded into $\mathcal{H}_{\nu, s}^{\infty}$. Using these results we prove, in Lemma 5.5 that $F \rightarrow H_{F}^{s}$ is bounded from $\mathcal{H}_{\nu, s}^{1}$ into $\mathcal{S}_{1}$. Finally, in Lemma [5.6 we find a bounded mapping $\tilde{\mathcal{T}}_{s}$ from the trace class $\mathcal{S}_{1}$ into $\mathcal{H}_{\nu, s}^{1}$ such that $\tilde{\mathcal{T}}_{s}\left(H_{F}^{s}\right)=F$.

Lemma 5.3. If $F \in \mathcal{H}_{\nu, s}^{1}$, then $H_{F}^{s} \in \mathcal{S}_{1}$.

Proof. Let $F \in \mathcal{H}_{\nu, s}^{1}$. By Theorem 4.1, $F=\sum_{j=1}^{\infty} F_{j}$ where

$$
F_{j}(w)=\left(1-\left|z_{j}\right|^{2}\right)^{\nu} K_{\nu, s}\left(w, z_{j}\right) \otimes^{s} B^{t}\left(z_{j}, z_{j}\right)^{1 / 2} a_{j}
$$

for some $\left\{z_{j}\right\}_{j=1}^{\infty} \subset \mathbb{B}$ and some $\left\{a_{j}\right\}_{j=1}^{\infty} \in l^{1}\left(\mathbb{B}, \odot^{s} V^{\prime}\right)$. We claim that

$$
\operatorname{rank} H_{F_{j}}^{s} \leq M_{s} \quad \text { for all } j=1,2,3, \ldots,
$$

where $M_{s}$ depends only on $s$ and $d$. Accepting temporarily the claim and using Theorem 1.1(a) in $\mathrm{Su}$ we get that

$$
\left\|H_{F}^{s}\right\|_{\mathcal{S}_{1}} \leq \sum_{j=1}^{\infty}\left\|H_{F_{j}}^{s}\right\|_{\mathcal{S}_{1}} \leq M_{s} \sum_{j=1}^{\infty}\left\|H_{F_{j}}^{s}\right\|_{\mathcal{S}_{\infty}} \leq M_{s}^{\prime} \sum_{j=1}^{\infty}\left\|F_{j}\right\|_{\nu, s, \infty} \leq M_{s}^{\prime \prime} \sum_{j=1}^{\infty}\left\|a_{j}\right\| .
$$

Now we go back to claim (5.1). By Lemma 2.7, $\mathcal{T}_{s}(f, g) \in \mathcal{H}_{\nu, s}^{1}$ for all $f, g \in L_{a}^{2}\left(d \iota_{\nu}\right)$. Thus, by the reproducing property in Theorem 2.1

$$
H_{F_{j}}^{s}(f, g)=c\left\langle\mathcal{T}_{s}(f, g)\left(z_{j}\right),\left(1-\left|z_{j}\right|^{2}\right)^{\nu} \otimes^{s} B^{t}\left(z_{j}, z_{j}\right)^{1 / 2} a_{j}\right\rangle .
$$

Fix $z_{0} \in \mathbb{B}$. Then $\mathcal{T}_{s}(f, g)\left(z_{0}\right)$ is a sum of finitely many rank one forms where the number $M_{s}$ of summands depends only on $s$ and $d$. To see this, we consider $f\left(z_{0}\right)=\left\langle f, K_{z_{0}}\right\rangle_{\nu}$. Since

$$
\partial^{s-k} f\left(z_{0}\right) \otimes \partial^{k} g\left(z_{0}\right)=\left\langle f, \overline{\partial^{s-k}} K_{z_{0}}\right\rangle_{\nu} \otimes\left\langle g, \overline{\partial^{k}} K_{z_{0}}\right\rangle_{\nu},
$$

then $(f, g) \rightarrow \partial^{s-k} f\left(z_{0}\right) \otimes \partial^{k} g\left(z_{0}\right)$ is a rank one form. Thus, the bilinear form $(f, g) \rightarrow \mathcal{T}_{s}(f, g)\left(z_{0}\right)$ has rank at most $M_{s}$ and so has $H_{F_{j}}$.

Lemma 5.4. The operator $\mathcal{I}: \mathcal{H}_{\nu, s}^{1} \rightarrow \mathcal{H}_{\nu, s}^{\infty}, \mathcal{I}(F)=F$, is bounded.

Proof. First, let $F \in \mathcal{H}_{\nu, s}^{1}$. Then $H_{F}^{s} \in \mathcal{S}_{1}$ by Lemma 5.3. Hence $H_{F}^{s} \in \mathcal{S}_{\infty}$, so by Theorem 1.1(a) in [Su it follows that $F \in \mathcal{H}_{\nu, s}^{\infty}$. Thus $\mathcal{I}$ is well-defined.

Now, assume that $F_{n} \rightarrow F$ in $\mathcal{H}_{\nu, s}^{1}$ and that $\mathcal{I}\left(F_{n}\right) \rightarrow G$ in $\mathcal{H}_{\nu, s}^{\infty}$. We shall prove that $\mathcal{I}(F)=G$. On the one hand, since $F_{n} \rightarrow F$ in $\mathcal{H}_{\nu, s}^{1}$, then there is a subsequence $\mathcal{I}\left(F_{n_{j}}\right)$ converging pointwise to $\mathcal{I}(F)$. On the other hand, since $\mathcal{I}\left(F_{n}\right) \rightarrow G$ in $\mathcal{H}_{\nu, s}^{\infty}$, then $\mathcal{I}\left(F_{n_{j}}\right) \rightarrow G$ pointwise. Thus $\mathcal{I}(F)=G$ and the operator $\mathcal{I}$ is bounded by the Closed Graph Theorem.

Lemma 5.5. The operator $\Gamma: \mathcal{H}_{\nu, s}^{1} \rightarrow \mathcal{S}_{1}, \Gamma(F)=H_{F}^{s}$, is bounded.

Proof. The operator $\Gamma$ is well defined by Lemma 5.3. We use the Closed Graph Theorem. Assume that $F_{n} \rightarrow F$ in $\mathcal{H}_{\nu, s}^{1}$ and that $\Gamma\left(F_{n}\right) \rightarrow B$ in $\mathcal{S}_{1}$. We shall prove that $H_{F}^{s}=B$. On the one hand, by Theorem 1.1(a) in [Su and Lemma 5.4 it follows that

$$
\left\|H_{F_{n}-F}^{s}\right\|_{\mathcal{S}_{\infty}} \leq C\left\|F_{n}-F\right\|_{\nu, s, \infty} \leq C^{\prime}\left\|F_{n}-F\right\|_{\nu, s, 1}
$$


so that $H_{F_{n}}^{s} \rightarrow H_{F}^{s}$ in $\mathcal{S}_{\infty}$. On the other hand,

$$
\left\|\Gamma\left(F_{n}\right)-B\right\|_{\mathcal{S}_{\infty}} \leq\left\|\Gamma\left(F_{n}\right)-B\right\|_{\mathcal{S}_{1}}
$$

so that $H_{F_{n}}^{s} \rightarrow B$ in $\mathcal{S}_{\infty}$. Thus $H_{F}^{s}=B$ so that $\Gamma$ has the closed graph property. Hence, $\Gamma$ is bounded.

We recall the transvectant $\tilde{\mathcal{T}}_{s}: \mathcal{S}_{\infty}\left(L_{a}^{2}\left(d \iota_{\nu}\right), L_{a}^{2}\left(d \iota_{\nu}\right)\right) \rightarrow \mathcal{A}_{s}(\mathbb{B} \times \mathbb{B})$ defined in $[\mathrm{Su}$ (see also $[\mathrm{FR}]$ and $[\mathrm{PZ}]$ ), where $\mathcal{A}_{s}(\mathbb{B} \times \mathbb{B})$ consists of all holomorphic functions $G: \mathbb{B} \times \mathbb{B} \rightarrow \odot^{s} V^{\prime}$. We recall further that the transvectant $\mathcal{T}_{s}$ in (2.10) can be defined for any holomorphic function $G(z, w)$ on $\mathbb{B} \times \mathbb{B}$, namely

$$
\left(\mathcal{T}_{s} G\right)(z, w)=\sum_{k=0}^{s}\left(\begin{array}{l}
s \\
k
\end{array}\right)(-1)^{k} \frac{\partial_{z}^{k} \odot \partial_{w}^{s-k} G(z, w)}{(\nu)_{k}(\nu)_{s-k}} .
$$

For bounded bilinear forms $A$ on $L_{a}^{2}\left(d \iota_{\nu}\right)$, we define

$$
\tilde{\mathcal{T}}_{s}(A)(z)=\left(\mathcal{T}_{s} G\right)(z, z),
$$

where $G(z, w)=\overline{A\left(K_{z}, K_{w}\right)}$.

Lemma 5.6. The operator $\tilde{\mathcal{T}}_{s}: \mathcal{S}_{1} \rightarrow \mathcal{H}_{\nu, s}^{1}$ defined in (5.2) is bounded. Also, $\tilde{\mathcal{T}}_{s}\left(H_{F}^{s}\right)=F$ if $H_{F}^{s} \in \mathcal{S}_{1}$.

Proof. First, let $B \in \mathcal{S}_{1}$ be of rank one. Then there exists $\phi, \varphi \in L_{a}^{2}\left(d \iota_{\nu}\right)$ such that

$$
B(f, g)=\langle f, \phi\rangle_{\nu}\langle g, \varphi\rangle_{\nu}
$$

for all $f, g \in L_{a}^{2}\left(d \iota_{\nu}\right)$. Then $\|B\|_{\mathcal{S}_{1}}=\|\phi\|_{\nu}\|\varphi\|_{\nu}$ and $\tilde{\mathcal{T}}_{s}(B)(z)=\mathcal{T}_{s}(\phi, \varphi)(z)$, so by Lemma 2.7 it follows that

$$
\left\|\tilde{\mathcal{T}}_{s}(B)\right\|_{\nu, s, 1} \leq C_{s}\|\phi\|_{\nu}\|\varphi\|_{\nu} \leq C_{s}\|B\|_{\mathcal{S}_{1}}
$$

In general, if $B \in \mathcal{S}_{1}$ we can write $B=\sum_{n=1}^{\infty} B_{n}$, $\operatorname{rank} B_{n}=1$ such that

$$
\left\|B^{N}\right\|_{\mathcal{S}_{1}}=\sum_{n=1}^{N}\left\|B_{n}\right\|_{\mathcal{S}_{1}} \rightarrow\|B\|_{\mathcal{S}_{1}}, \text { as } N \rightarrow \infty
$$

where $B^{N}=\sum_{n=1}^{N} B_{n}$. By (5.3) the sequence $\left\{\tilde{\mathcal{T}}_{s}\left(B^{N}\right)\right\}_{n=1}^{\infty}$ is Cauchy and hence converges to some $G$ in $\mathcal{H}_{\nu, s}^{1}$. Now, since $B^{N} \rightarrow B$ in $\mathcal{S}_{\infty}$ it follows by Lemma 8.4 in $\left[\mathrm{Su}\right.$ that $\tilde{\mathcal{T}}_{s}\left(B^{N}\right) \rightarrow \tilde{\mathcal{T}}_{s}(B)$ in $\mathcal{H}_{\nu, s}^{\infty}$. Hence $\tilde{\mathcal{T}}_{s}(B)=G$ so that (5.3) holds for any $B \in \mathcal{S}_{1}$.

Also, if $H_{F}^{s} \in \mathcal{S}_{1}$, then $\tilde{\mathcal{T}}_{s}\left(H_{F}^{s}\right)=F$. (As in the proof of Theorem 5.2 we refer to the proof of Lemma 8.6 in $[\mathrm{Su}$.)

\section{REFERENCES}

[FR] S. Ferguson and R. Rochberg, Higher order Hilbert-Schmidt Hankel forms and tensors of analytic kernels, Math. Scand. 96 (2005), no. 1, 117-146. MR2142876 (2006b:47038)

[HKZ] H. Hedenmalm, B. Korenblum, K. Zhu, Theory of Bergman spaces, Springer-Verlag, New York-Berlin-Heidelberg, 2000. MR.1758653 (2001c:46043)

[JP] S. Janson and J. Peetre, A new generalization of Hankel operators (the case of higher weights), Math. Nachr. 132 (1987), 313-328. MR0910059 (88m:47045)

[JPR] S. Janson, J. Peetre and R. Rochberg, Hankel forms and the Fock space, Revista Mat. Iberoamer. 3 (1986), 61-138. MR1008445 (91a:47029)

[L] O. Loos, Bounded symmetric domains and Jordan pairs, The University of California at Irvine, 1977.

[P1] J. Peetre, Hankel kernels of higher weight for the ball, Nagoya Math. J. 130 (1993), 183192. MR $1223736(94 \mathrm{i}: 32005)$ 
[P2] J. Peetre, Hankel forms of arbitrary weight over a symmetric domain via the transvectant, Rocky Mountain J. Math. 24 (1994), no. 3, 1065-1085. MR.1307592 (96f:47051)

[Pe1] V. V. Peller, Hankel operators and their applications, Springer-Verlag, 2002. MR.1949210 (2004e:47040)

[Pe2] V. V. Peller, Vectorial Hankel operators, commutators and related operators of the Schatten-von Neumann class $\gamma_{p}$, Integral Equations Operator Theory 5 (1982), no. 2, 244-272. MR0647702 (83f:47024)

[PZ] L. Peng and G. Zhang, Tensor product of holomorphic representations and bilinear differential operators, J. Funct. Anal. 210 (2004), no. 1, 171-192. MR.2052118 (2004m:22024)

[RS] M. Reed and B. Simon, Methods of modern mathematical physics I: Functional analysis, Academic press, 1980. MR0751959 (85e:46002)

[Ro] H. Rosengren, Multilinear Hankel forms of higher order and orthogonal polynomials, Math. Scand. 82 (1998), 53-88. MR1634657 (99m:47033)

[Ru] W. Rudin, Function theory in the unit ball of $\mathbb{C}^{n}$, Springer-Verlag, 1980. MR0601594 (82i:32002)

[S] B. Simon, Trace ideals and their applications, Cambridge University Press, CambridgeLondon-New York-Melbourne, 1979. MR0541149 (80k:47048)

[Su] M. Sundhäll, Schatten-von Neumann properties of bilinear Hankel forms of higher weights, Math. Scand. 98 (2006), 283-319.

[Z] G. Zhang, Tensor products of weighted Bergman spaces and invariant Ha-plitz operators, Math. Scand. 71 (1992), no. 1, 85-95. MR1216104 (94e:47039a)

Department of Mathematics, Chalmers University of Technology and Göteborg UniVERsity, SE-412 96 Göteborg, Sweden

E-mail address: sundhall@math.chalmers.se 